\title{
Mechanical relaxation in glasses and at the glass transition
}

\author{
U. Buchenau \\ Institut für Festkörperforschung, Forschungszentrum Jülich, Postfach 1913, D-52425 Jülich, Germany
}

(Received 18 August 2000; published 14 February 2001)

\begin{abstract}
The Gilroy-Phillips model of relaxational jumps in asymmetric double-well potentials, developed for the Arrhenius-type secondary relaxations of the glass phase, is extended to a formal description of the breakdown of the shear modulus at the glass transition, the $\alpha$ process. The extension requires the introduction of two separate parts of the barrier distribution function $f(V)$, with a different temperature behavior of primary and secondary parts, respectively. The time-temperature scaling of the $\alpha$ process, together with a sum rule for the whole barrier distribution function, implies a strong rise of the integrated secondary relaxation with increasing temperature above the glass transition. Thus one gets a quantitative relation between the fragility of the glass former and the fast rise of the picosecond process observed in neutron and Raman scattering. The formalism is applied to literature data of polystyrene, vitreous silica and a sodium silicate glass. In the glass phase of polystyrene, one finds a temperature-independent secondary barrier distribution function, in agreement with an earlier Raman result from the literature. Above the glass transition, the secondary barrier distribution function increases with temperature as predicted. The findings allow for an interpretation of the fragility and the entropy crisis at the glass transition.
\end{abstract}

DOI: 10.1103/PhysRevB.63.104203

PACS number(s): 63.50.+x, 64.70.Pf

\section{INTRODUCTION}

Relaxation in glasses, sometimes also called secondary relaxation to distinguish it from the primary relaxation at the glass transition, is generally believed ${ }^{1-3}$ to be well described in terms of the Arrhenius-Kramers picture, ${ }^{4}$ with a relaxation time $\tau_{V}$ given by the Arrhenius relation

$$
\tau_{V}=\tau_{0} \mathrm{e}^{V / k_{B} T},
$$

where $\tau_{0}$ is a microscopic time of the order of $10^{-13}$ seconds, $V$ is the energy of the barrier between two energy minima of the system, and $T$ is the temperature.

In contrast, the primary relaxation or $\alpha$-process, the onset of the flow process at the glass transition temperature $T_{g}$ and above, seems to follow a much steeper law ${ }^{5,6}$

$$
\tau_{\alpha}=\tau_{0} \mathrm{e}^{A /\left(T-T_{0}\right)},
$$

where $A$ and $T_{0}$ are constants with the dimension of a temperature. This is the well-known empirical Vogel-FulcherTammann (VFT) or Williams-Landel-Ferry (WLF) equation. $T_{0}$, the Vogel-Fulcher temperature, is smaller than $T_{g}$; the closer it lies to $T_{g}$, the more fragile is the glass former.

Since the Arrhenius law has a sound microscopic background $^{4}$ and the VFT or WLF equation has not, it seems reasonable to build a joint quantitative description on the former, bearing in mind the physical difference of the two processes. This is the intention of the present paper.

In glasses, one has to reckon with a whole distribution of relaxational jumps, not only over different potential barrier heights, but also between energy minima of different energy. Thus one has to extend the classical Arrhenius-Kramers treatment ${ }^{4}$ of a thermally activated relaxation process in a symmetric double-well potential to deal with a broad distribution of barrier heights and asymmetries. A distribution in the barrier heights was considered by Fröhlich, ${ }^{7}$ but only for symmetric potentials. An asymmetric multiminimum situa- tion was solved by Hoffman and Pfeiffer. ${ }^{8}$ But none of these early attempts dealt simultaneously with a broad distribution in both quantities, the barrier height, and the asymmetry of the wells. The necessity of such a double distribution was recognized in the tunneling model ${ }^{9}$ for the two-level states below $1 \mathrm{~K}$ in 1972. The tunneling model was a major breakthrough; in the same year, Pollak and Pike ${ }^{10}$ applied the double distribution concept to classical relaxation in order to explain the ac conductivity of glasses. Nine years later, Gilroy and Phillips ${ }^{11}$ extended the scheme to a general description of mechanical and dielectric relaxation processes in glasses at higher temperatures. Also, they drew a parallel between mechanical relaxation data and the quasielastic part of the Raman scattering. At the end of the same decade, the soft-potential model postulated a relation between the tunneling states and the low barrier classical relaxation processes in glasses (an excellent review of the soft-potential model has been given by Parshin ${ }^{12}$ ). Nevertheless, up to now only a few checks of these postulates for dynamical mechanical, ${ }^{11,13-15}$ Raman $^{11,16,17}$ and neutron ${ }^{18}$ data have been reported in the literature.

The present paper begins in Sec. II with a discussion of the Gilroy-Phillips model, and a derivation of its connection to rheology. It turns out that one can define a barrier distribution function $f(V)$ to describe the mechanical shear relaxation at different temperatures and frequencies. The integral of this barrier distribution function over all barrier heights $V$ must equal 1 to bring the shear modulus down to zero. In order to include the flow process into the same scheme, one separates $f(V)$ into two parts, $f_{s}(V)$ and $f_{\alpha}(V)$. The first of these describes the secondary relaxations in the glass phase, the second describes the $\alpha$ process in the undercooled liquid, respectively. As will be seen, the sum rule for the total barrier distribution function supplies a quantitative basis for Angell's conjecture ${ }^{19}$ of a relation between the fragility and the rise of the fast picosecond process above the glass transition. 


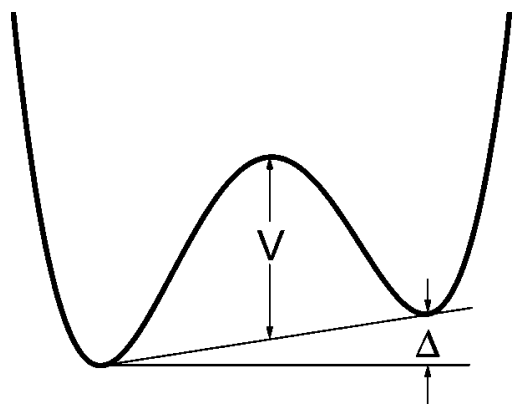

FIG. 1. Asymmetric double-well potential with barrier height $V$ and asymmetry $\Delta$ as a function of a generalized coordinate.

The low-barrier part of $f_{s}(V)$ determines the relaxational part of neutron and Raman scattering. The corresponding equations are derived.

Section III applies the equations to determine $f(V)$ from literature data for amorphous polystyrene, vitreous silica, and a sodium silicate glass. From the results, one gets an impression as to whether one gets the same secondary barrier distribution function from different methods, in particular if one compares the high-frequency neutron, Raman, and Brillouin scattering results with the low-frequency torsion pendulum or creep data. Furthermore, one gets a feeling for the amount of reduction of the shear modulus by the secondary relaxation processes in the glass phase. As we will see, the findings suggest a generalized Maxwell criterion for the onset of the glass transition, namely that the flow begins when the shear response from the secondary relaxation equals the elastic one. Section IV compiles and discusses these results and their possible significance for our view of the glass transition. Summary and conclusions are given in Sec. V.

\section{THE GILROY-PHILLIPS MODEL}

\section{A. The asymmetric double-well potential}

Let us denote the shear strain by $\epsilon$, the shear stress by $\sigma$ and the (infinite frequency) shear modulus by $G$. $G$ will generally depend on the temperature $T$.

The structural relaxation is taken to be a superposition of independent Debye relaxation centers in asymmetric doublewell potentials with two minima, as shown in Fig. 1. The energy of the left minimum is $-\Delta / 2$ and the energy of the right minimum is $+\Delta / 2$. The height of the barrier is $V$.

The interaction between the shear strain and the Debye relaxation center is described by the change of the asymmetry under the influence of the strain. The interaction is characterized by the coupling parameter $\gamma$, leading to an asymmetry $\Delta+\gamma \epsilon$ of the relaxation in the strained glass. $\gamma$ must be considered to depend both on $V$ and $\Delta$.

The free energy $F$ of the relaxation center reads

$$
F=-k_{B} T \ln \left[2 \cosh \left(\frac{\Delta+\gamma \epsilon}{2 k_{B} T}\right)\right]
$$

which has the second derivative with respect to the shear distortion $\epsilon$

$$
\frac{\partial^{2} F}{\partial \epsilon^{2}}=-\frac{\gamma^{2}}{4 k_{B} T \cosh ^{2}\left(\Delta / 2 k_{B} T\right)} .
$$

The second derivative determines the contribution of that specific relaxing entity to the difference between the shear moduli at infinite and zero frequency. The equation shows that the main influence on the shear modulus is due to relaxation in potentials with asymmetries smaller than $k_{B} T$; for larger asymmetries the influence decreases rapidly because of the square of the hyperbolic cosine in the denominator.

\section{B. The barrier distribution function $f(V)$}

We want to calculate the frequency dependence of the shear modulus under the assumption of slowly varying distribution functions in the parameters $V$ and $\Delta$. In detail, we assume a number density of relaxing entities $n(V, \Delta)$ and a coupling constant $\gamma(V, \Delta)$ which are both approximately constant if either $V$ or $\Delta$ is varied by an amount of the order of the thermal energy $k_{B} T$.

Under this assumption, it is safe to neglect as well the influence of the asymmetry on the relaxation time. We assume the relaxation time $\tau_{V}$ to be given by the Arrhenius Eq. (1).

We then integrate over the asymmetry $\Delta$ to obtain the step $\delta G$ between the shear moduli at infinite and zero frequency from all relaxation centers with barrier heights between $V$ and $V+d V$

$$
\delta G=d V \int_{-\infty}^{\infty} \frac{\gamma^{2} n(V, \Delta) d \Delta}{4 k_{B} T \cosh ^{2}\left(\Delta / 2 k_{B} T\right)} .
$$

Since one has only contributions in the near neighborhood of $\Delta=0$, where $n(V, \Delta) \approx n(V, 0)$, and since

$$
\int_{-\infty}^{\infty} \frac{d \Delta}{\cosh ^{2}\left(\Delta / 2 k_{B} T\right)}=4 k_{B} T
$$

one finds

$$
\delta G=\gamma^{2} n(V, 0) d V
$$

This is different from a single relaxation in a symmetric potential, where the step in the modulus is inversely proportional to the temperature. The physical reason for this difference is clear: As the temperature rises, relaxation centers with higher and higher asymmetry begin to contribute to the step in the modulus. This is an important difference between relaxation in crystals and relaxation in disordered matter.

The temperature-independent step in the modulus is determined by the barrier distribution function $f(V)$, defined by

$$
f(V)=\frac{\gamma^{2} n(V, 0)}{G} .
$$

This parameter combination can be argued to remain independent of temperature, even if $G$ varies with temperature, considering the relaxing entity as a small misfit region in an elastic medium, ${ }^{20}$ a misfit region which is able to change the 
sign of the misfit by jumping over the barrier. Here, however, this argument will not be given in detail.

The frequency dependence of the complex shear modulus at the frequency $\omega$ and the temperature $T$ reads

$$
\begin{gathered}
\frac{G^{\prime}(\omega, T)}{G}=\frac{G_{e}}{G}+\int_{0}^{\infty} f(V) \frac{\omega^{2} \tau_{V}^{2} d V}{1+\omega^{2} \tau_{V}^{2}} \\
\frac{G^{\prime \prime}(\omega, T)}{G}=\int_{0}^{\infty} f(V) \frac{\omega \tau_{V} d V}{1+\omega^{2} \tau_{V}^{2}},
\end{gathered}
$$

where $\tau_{V}$ is a function of $V$ by the Arrhenius relation Eq. (1), and $G_{e}$ is the zero frequency modulus after the decay of all the relaxations in the system.

These two equations describe the real and the imaginary part of the frequency-dependent shear modulus at all frequencies and temperatures. As long as one can reckon with a temperature-independent number of uncoupled relaxation centers, the barrier distribution function $f(V)$ remains temperature independent.

But Eqs. (9) and (10) have a much higher potential than a simple description of uncoupled relaxation centers in disordered matter. If one allows for a temperature dependence of the barrier distribution function $f(V)$, one can describe any relaxational behavior. In particular, it is possible to obtain a unified picture of the relaxational properties of the glass and the high-viscosity flow of the undercooled liquid. This is the central point of the present paper.

As shown in the next subsection, one can rewrite the conventional rheological expressions ${ }^{21}$ in terms of $f(V)$. The advantage of the choice of $f(V)$ lies in the possibility to distinguish the trivial Arrhenius temperature dependence from other, nontrivial temperature changes. These nontrivial temperature changes will then reflect in a temperature dependence of $f(V)$.

\section{Rheological equations in terms of $f(V)$}

Comparing the two expressions, Eqs. (9) and (10), to those in the textbooks, for instance the one on polymers by Ferry ${ }^{21}$ [Chap. 3, Eqs. (23) and (24)], one finds the relation between the rheological relaxation function $H(\tau)$ and the barrier distribution function $f(V)$

$$
H\left(\tau_{0} \mathrm{e}^{V / k_{B} T}\right)=H\left(\tau_{V}\right)=G k_{B} T f(V) .
$$

With this equation, one can rewrite all the exact and approximate rheological relations ${ }^{21}$ in terms of $f(V)$. To do this, one first has to define a convenient equivalent function $l(V)$ to the conventional rheological function $L(\tau)$, which is needed whenever one wants to calculate a compliance

$$
L\left(\tau_{0} \mathrm{e}^{V / k_{B} T}\right)=L\left(\tau_{V}\right)=\frac{k_{B} T l(V)}{G} .
$$

In the following, the most important equations of chapter 3 of Ferry's book ${ }^{21}$ are translated into the Gilroy-Phillips notation. Ferry's Eq. (19) for the time-dependent modulus $G(t)$ reads

$$
G(t)=G_{e}+\int_{-\infty}^{\infty} H(\tau) \mathrm{e}^{-t / \tau} d \ln \tau
$$

and translates into

$$
G(t)=G_{e}+G \int_{0}^{\infty} f(V) \mathrm{e}^{-t / \tau_{V}} d V
$$

For a viscoelastic liquid, the zero frequency modulus $G_{e}$ $=0$, so one must have

$$
\int_{0}^{\infty} f(V) d V=1 .
$$

This is the sum rule for the barrier distribution function $f(V)$. It has important consequences for the connection between primary and secondary relaxation, as discussed in the next subsection.

Next, there is Ferry's Eq. (20) for the compliance

$$
J(t)=\frac{1}{G}+\int_{-\infty}^{\infty} L(\tau)\left(1-\mathrm{e}^{-t / \tau}\right) d \ln \tau+\frac{t}{\eta_{0}},
$$

where $\eta_{0}$ is the viscosity, which translates into

$$
J(t)=\frac{1}{G}\left[1+\int_{0}^{\infty} l(V)\left(1-\mathrm{e}^{-t / \tau_{V}}\right) d V\right]+\frac{t}{\eta_{0}} .
$$

The viscosity $\eta_{0}$ can be calculated from Ferry's Eq. (28)

$$
\eta_{0}=\int_{-\infty}^{\infty} \tau H(\tau) d \ln \tau,
$$

which translates into

$$
\eta_{0}=G \int_{0}^{\infty} \tau_{V} f(V) d V .
$$

Ferry's Eqs. (21) and (22), the transformation from $H(\tau)$ to $L(\tau)$ and back, read

$$
L=\frac{H}{\left[G_{e}-\int_{-\infty}^{\infty} \frac{H(u)}{\tau / u-1} d \ln u\right]^{2}+\pi^{2} H^{2}}
$$

and

$$
H=\frac{L}{\left[\frac{1}{G}+\int_{-\infty}^{\infty} \frac{L(u)}{1-u / \tau} d \ln u-\frac{\tau}{\eta_{0}}\right]^{2}+\pi^{2} L^{2}}
$$

They translate into

$$
l(V)=\frac{f(V)}{\left[\frac{G_{e}}{G}-I_{f}(V)\right]^{2}+\left[\pi k_{B} T f(V)\right]^{2}}
$$

with 


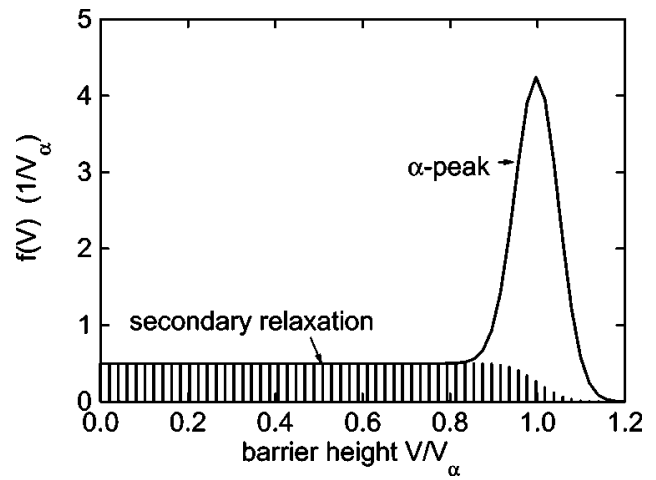

FIG. 2. Secondary barrier distribution function $f_{s}(V)$ (the shaded area), together with the cutoff by the $\alpha$-relaxation peak (schematic).

$$
I_{f}(V)=\int_{0}^{\infty} \frac{f(E) d E}{\exp \left[(V-E) / k_{B} T\right]-1}
$$

and

$$
f(V)=\frac{l(V)}{\left[1-\frac{\tau_{V} G}{\eta_{0}}+I_{l}(V)\right]^{2}+\left[\pi k_{B} T l(V)\right]^{2}}
$$

with

$$
I_{l}(V)=\int_{0}^{\infty} \frac{l(E) d E}{1-\exp \left[(E-V) / k_{B} T\right]} .
$$

With these exact equations, one can calculate the mechanical response for any type of shear experiment for a given barrier distribution function $f(V)$. The reverse, the determination of $f(V)$ from experimental data, is more difficult, because the exact equations are integral equations. Nevertheless, one can start to determine a first approximation to $f(V)$ from measurements of $G^{\prime}$ and $G^{\prime \prime}$ using the crude approximations

$$
\frac{G^{\prime}}{G}=1-\int_{0}^{k_{B} T \ln \left(1 / \omega \tau_{0}\right)} f(V) d V
$$

and

$$
f\left[k_{B} T \ln \left(1 / \omega \tau_{0}\right)\right]=\frac{2}{\pi} \frac{G^{\prime \prime}}{G k_{B} T} .
$$

\section{Primary and secondary relaxation}

It is quite clear that one needs to distinguish secondary and primary processes, because their physical mechanism is different. Thus one has to distinguish between $f_{s}(V)$, the secondary barrier distribution function of the secondary Arrhenius relaxation, and $f_{\alpha}(V)$, the primary barrier distribution function for the primary $\alpha$ process or flow process (see Fig. 2).

For many glass formers, in particular polymeric ones, ${ }^{21}$ $H_{\alpha}\left(\tau / \tau_{\alpha}\right)$ is independent of the temperature. This is the time-temperature scaling of the $\alpha$ process, sometimes also denoted as thermorheological simplicity. Since the factor $G k_{B} T$ in Eq. (11) between $H(\tau)$ and $f(V)$ varies only weakly with temperature, this implies that the primary barrier distribution function $f_{\alpha}(V)$ is an essentially temperatureindependent function of $V-V_{\alpha}(T)$, where $V_{\alpha}(T)$ denotes the maximum of this strongly peaked function. From the Vogel-Fulcher law Eq. (2), one expects the temperature dependence

$$
V_{\alpha}(T)=V_{\alpha}\left(T_{g}\right) \frac{T\left(T_{g}-T_{0}\right)}{T_{g}\left(T-T_{0}\right)},
$$

showing the divergence of the fictive Arrhenius barrier of the flow process towards the Vogel-Fulcher temperature $T_{0}$.

If time-temperature scaling holds, the weight $w_{\alpha}$ of the $\alpha$ process, given by

$$
w_{\alpha}=\int_{0}^{\infty} f_{\alpha}(V) d V
$$

should be temperature independent. In the comparison to experiment, we will see that $w_{\alpha}$ tends to be close to $1 / 2$.

What does this imply for the secondary relaxations? The $\alpha$ process is also an upper cutoff for the secondary relaxation; at the end of the process, the long-time shear modulus is zero. If a secondary relaxation barrier is too high, the relaxing entity will flow away before it has a chance to jump. Therefore there is a steep cutoff for the secondary barrier distribution function $f_{s}(V)$ at $V_{\alpha}(T)$. According to the sum rule Eq. (15)

$$
\int_{0}^{V_{\alpha}(T)} f_{s}(V) d V=1-w_{\alpha}=\text { const } \approx \frac{1}{2} .
$$

With this sum rule, the decrease of $V_{\alpha}(T)$ with increasing temperature implies that the secondary barrier distribution function $f_{s}(V)$ must increase with increasing temperature. This increase will be stronger for more fragile glass formers. Such a connection between the fragility and the rise of the fast process above $T_{g}$ has been indeed postulated empirically; ${ }^{19}$ here we will be able to quantify this connection.

The increase of $f_{s}(V)$ above the glass temperature can be characterized to first order by

$$
f_{s}(V)=f_{s}\left(V, T_{g}\right)\left[1+\alpha_{s}(V) \frac{T-T_{g}}{T_{g}}\right] .
$$

One can define an average temperature coefficient $\bar{\alpha}_{s}$ by

$$
\bar{\alpha}_{s}=\frac{1}{1-w_{\alpha}} \int_{0}^{V_{\alpha}\left(T_{g}\right)} \alpha_{s}(V) f_{s}(V) d V
$$

Differentiating the sum rule Eq. (30) for $f_{s}(V)$ with respect to the temperature, calculating the derivative of $V_{\alpha}(T)$ with respect to temperature from Eq. (28) and using Eq. (32), one finds the relation between the fragility and the average rise of the secondary relaxation 


$$
\frac{T_{0}}{T_{g}-T_{0}}=\frac{\bar{\alpha}_{s}\left(1-w_{\alpha}\right)}{V_{\alpha}\left(T_{g}\right) f_{s}\left[V_{\alpha}\left(T_{g}\right)\right]} .
$$

This relation will become clearer in the next subsection, where the simplest possible case of a constant secondary barrier distribution function is discussed.

\section{E. The generic case $f_{s}(V)=$ const}

The deep implications of the Gilroy-Phillips formulation of the $\alpha$-process are more clearly seen in the simplest possible case, shown in Fig. 2. Let us assume $f_{s}(V)=$ const and $\alpha_{s}(V)=$ const. If the rise of $f_{s}(V)$ with temperature is strictly linear, Eqs. (30) and (31) imply

$$
V_{\alpha}(T)=\frac{V_{\alpha}\left(T_{g}\right) T_{g}}{T_{g}+\alpha_{s}\left(T-T_{g}\right)},
$$

which is not exactly equal, but very close to the empirical Vogel-Fulcher-Tammann or Williams-Landel-Ferry Eq. (2), with the Vogel-Fulcher temperature $T_{0}$ given by

$$
T_{0}=T_{g}\left(1-\frac{1}{\alpha_{s}}\right) \text {. }
$$

It is obvious how this comes about: at $T_{0}$, the density of secondary processes extrapolates to zero. Thus one has to proceed to infinitely high barriers to satisfy the sum rule (30). This gives a different view on the puzzling fragility of glass formers: the abnormal temperature dependence is a consequence of the time-temperature scaling of the $\alpha$ process, and of a strictly linear rise of the number of secondary relaxing units with temperature.

The decrease of the secondary barrier distribution function $f_{s}(V)$ implies a decrease of the number of minima of the glass former. Thus one gets an equality ${ }^{22}$ between the VogelFulcher and the Kauzmann temperature $T_{K}$, the latter being defined as the temperature where the excess entropy of the glass former over the corresponding crystalline system extrapolates to zero. If there are no minima between which the glass former can jump, there is no excess entropy, the old Adam-Gibbs idea. ${ }^{23}$

Note that Eq. (35) for the Vogel-Fulcher or Kauzmann temperature holds not only in the generic case of a constant secondary barrier distribution function, but for any $f_{s}(V)$, as long as one can reckon with the same strictly linear temperature rise of the function for all $V$.

To complete the discussion of the glass transition peculiarities $^{6}$ in the Gilroy-Phillips picture, let us look at the stretching of the $\alpha$ process, empirically described by the Kohlrausch equation

$$
G(t)=G_{\alpha} \exp \left[-\left(t / \tau_{\alpha}\right)^{\beta}\right],
$$

where the Kohlrausch exponent $\beta$ lies $^{5}$ between 0.3 and 0.7 , and $G_{\alpha}$ is a free parameter. The smaller $\beta$ is, the more stretched is the $\alpha$ relaxation, and the stronger it deviates from a simple exponential decay.

In order to calculate $\beta$, the definition of the primary barrier distribution function $f_{\alpha}(V)$ must be more specific. Let us assume a Gaussian centered at $V_{\alpha}$ with weight $w_{\alpha}=1 / 2$ and with a full width at half maximum denoted by $\Delta_{\alpha}$. Calculating $G(t)$ from Eq. (13), one then finds fairly Kohlrauschlike curves for the simple generic case of Fig. 2, at least in the time region of the $\alpha$ process, with $G_{\alpha}$ $\approx 0.55 \ldots 0.75 G$ and $\tau_{\alpha}$ about $80 \%$ of the Arrhenius value for $V_{\alpha}$. There is a deviation of the Kohlrausch fits from the calculated curves, but it is so small that it would be hard to see in an experiment. It turns out that the ratio $\Delta_{\alpha} / V_{\alpha}$ determines the Kohlrausch exponent $\beta$; if it is 0.05 , then $\beta \approx 0.7$; for the ratio $0.1, \beta \approx 0.5$ and for the ratio $0.2, \beta \approx 0.3$. So the broader $f_{\alpha}(V)$, the more stretched the relaxation, not unexpected.

Note this is merely a change of description. The GilroyPhillips formulation does not really explain the puzzling features of the glass transition, the fragility, the entropy crisis and the stretching. But it supplies a description which allows to look for another explanation. We will return to this point in the discussion.

In order to measure $\alpha_{s}(V)$ in the picosecond range by scattering methods, one still needs the equations for the neutron- and Raman-scattering functions in terms of the barrier distribution function. These will be derived in the next subsection, the last part of the description of the GilroyPhillips model.

\section{F. Neutron and Raman scattering}

One can carry out the same integrations over asymmetries and barrier heights as in the shear relaxation for the neutronscattering cross section. Let us begin with a single asymmetric double-well potential, let us assume that atom $j$ has a coherent scattering length $b_{j}$ and an incoherent scattering cross section $\sigma_{j}$, and that it jumps from the position $-\vec{d}_{j} / 2$ to $\vec{d}_{j} / 2$, with the origin of the coordinate system in the middle between the two.

For the incoherent inelastic scattering in the one-phonon approximation, ${ }^{24}$ it suffices to calculate the mean-square displacements. These can be obtained from the Boltzmann occupation factors of the two minima of the potential. The average position vector $\vec{r}_{j}$ of atom $j$ is given by

$$
\left\langle\vec{r}_{j}\right\rangle=-\frac{\vec{d}_{j}}{2} \tanh \frac{\Delta}{2 k_{B} T}
$$

and its average square is

$$
\left\langle\vec{r}_{j}^{2}\right\rangle=\frac{d_{j}^{2}}{4}
$$

so the mean-square displacement contribution of the relaxation to atom $j$ reads

$$
\left\langle u_{j}^{2}\right\rangle=\left\langle\vec{r}_{j}^{2}\right\rangle-\left\langle\vec{r}_{j}\right\rangle^{2}=\frac{d_{j}^{2}}{4 \cosh ^{2} \Delta / 2 k_{B} T} .
$$


In this expression, we recognize the same inverse $\cosh ^{2}$ as in Eq. (4), which can be again integrated over the asymmetries, if the jump eigenvector stays essentially the same for the different local asymmetries.

The scattering contribution is a Lorentzian with a half width at half maximum in $\omega$ given by the inverse relaxation time, determined by the barrier height according to the Arrhenius Eq. (1). The weight of the contribution in $S(Q, \omega)$ is determined by the number of relaxations and the weighted sum of the jump vectors

$$
d^{2}=\frac{\sum_{j} \sigma_{j} d_{j}^{2}}{\bar{\sigma}} .
$$

The sum is over all atoms in the sample, and $\bar{\sigma}$ is their average incoherent cross section.

Integrating over the barrier heights as well, one gets the equation for the incoherent scattering

$$
S_{i n c}(Q, \omega)=v_{a} n\left(k_{B} T \ln \frac{1}{\omega \tau_{0}}, 0\right) \frac{k_{B}^{2} T^{2} Q^{2} d^{2}}{6 \omega},
$$

where $v_{a}$ is the atomic volume, and the prefactor $1 / 3$ stems from the directional average. This equation is again an approximation, which holds if $f(V)$ does not vary strongly with $V$. The coherent scattering is obtained replacing $d^{2}$ by $d_{c o h}^{2}(Q)$ with

$$
d_{c o h}^{2}(Q)=\frac{3}{\bar{b}^{2} Q^{2}}\left\langle\left|\sum_{j} b_{j} \mathrm{e}^{i \vec{Q} \vec{R}_{j}} \vec{Q} \vec{d}_{j}\right|^{2}\right\rangle,
$$

where $\vec{R}_{j}$ is the equilibrium position of atom $j, \bar{b}$ is the average scattering length and the brackets indicate the orientational averaging over the structure factor of the relaxation. Even after this averaging, the structure factor need not show a simple $Q^{2}$ behavior like the incoherent one, but does still contain information on the jump vectors. ${ }^{18}$

The scattering measurements do not give $f(V)$, but rather the product $n(V, 0) d^{2}$. If one wants the proportionality factor between those two quantities, one needs additional information about the relaxing entities. However, there is an elegant and general way to obtain this proportionality factor for very low barriers from the soft-potential model, ${ }^{12}$ which describes the tunneling states and the low-barrier classical relaxation as similar modes with a double-well potential distribution. It is not very difficult to derive an equation for the barrier distribution function $f(V)$ as defined here in terms of the definitions in this paper. One finds

$$
f_{s p}(V)=\frac{2 C}{W^{3 / 4} V^{1 / 4}},
$$

where $C$ (in principle $C_{l}$ for longitudinal waves and $C_{t}$ for transverse waves) is a dimensionless constant of the order of $10^{-4}$, which can be taken from acoustic attenuation measurements below $4 \mathrm{~K}$, and $W$ is the crossover energy between tunneling and vibrational modes, which can be measured from the crossover regions of the specific heat or the thermal conductivity at low temperatures. ${ }^{15}$ The soft-potential model has a fourth parameter $P_{s}$ for the density of these modes. With this parameter, the relation between $n(V, 0) d^{2}$ and the secondary barrier distribution function $f_{s}(V)$ reads

$$
v_{a} n(V, 0) d^{2} \frac{2 W^{2} \rho C_{t}}{\hbar^{2} P_{s}}=f_{s}(V),
$$

where $\rho$ is the mass density. For glasses consisting of more than one sort of atoms, the relation might fail if strongly and weakly scattering atoms have different jump widths. But with this relation, one can determine the secondary barrier distribution function $f_{s}(V)$ from neutron scattering measurements without adaptable parameter, using soft-potential parameters from the literature. ${ }^{15}$

The Raman scattering from relaxations in glasses is not so easily calculated. However, experience ${ }^{25,26}$ shows that neutron and Raman scattering give the same spectra as long as one stays at frequencies well below the boson peak. Thus one can use the Raman spectra as one uses incoherent neutron scattering data, with the disadvantage of an additional general adaptable parameter for the overall intensity. The advantage of the Raman technique is a much higher intensity and a much better resolution, allowing one to assess much lower frequencies.

\section{COMPARISON TO EXPERIMENT}

\section{A. Values from different techniques}

Let us first consider which barriers one samples with a given technique. Creep measurements cover the time range from $0.1 \mathrm{~s}$ to several weeks. This is a measurement in the time domain, applied mostly to measurements of the $\alpha$ process at the glass transition, which does not obey the Arrhenius relation Eq. (1). Nevertheless, one can formally calculate barrier heights of the order of 27 to $41 k_{B} T_{g}$, around $1 \mathrm{eV}$ for polystyrene $\left(T_{g}=373 \mathrm{~K}\right)$ and around $4 \mathrm{eV}$ for vitreous silica $\left(T_{g}=1473 \mathrm{~K}\right)$.

The torsion pendulum method with frequencies around 1 $\mathrm{Hz}$ sees relaxations around $0.1 \mathrm{~s}$. In terms of the Arrhenius relation with $\tau_{0}=10^{-13} \mathrm{~s}$, this implies a barrier height of 50 $\mathrm{meV}$ at $20 \mathrm{~K}$. In order to see barriers of $1 \mathrm{eV}$, one needs a temperature of about $400 \mathrm{~K}$, close to the glass temperature of polystyrene.

Proceeding to higher frequencies, one has vibrating reed measurements around $10 \mathrm{kHz}$, ultrasonic data in the $\mathrm{MHz}$ range, light scattering Brillouin data around $10 \mathrm{GHz}$ and, finally, Raman and neutron data between a few $\mathrm{GHz}$ and a few hundred GHz. For the latter two, the lower limit holds only for the Raman technique; if one looks for the weak quasielastic scattering from secondary relaxations, the neutron technique in practice has a lower limit of $100 \mathrm{GHz}$. The upper limit of about $300 \mathrm{GHz}$ is given by the crossover from relaxational to vibrational scattering. ${ }^{16,35}$ Thus one sees only the uppermost frequency band of the relaxational scattering with neutrons. Nevertheless, neutrons play an important role, because they serve to validate the Raman scattering data.

For the fast relaxation at $200 \mathrm{GHz}$, the relaxation time is of the order of a picosecond, only ten times longer than the microscopic time scale of the vibrational motion. The 
Arrhenius relation translates this again into a barrier of 50 $\mathrm{meV}$ at $300 \mathrm{~K}$. Thus neutron and Raman measurements at room temperature sample the same relaxations that one sees in a torsion pendulum measurement around $20 \mathrm{~K}$. If one is still in the glass phase at room temperature, one can thus check the temperature independence of the barrier distribution function $f(V)$ [in that case, $f(V)=f_{s}(V)$ ] by a comparison between a torsion pendulum and a neutron or Raman experiment. Naturally, the same can be done by a comparison of a torsion pendulum and a Brillouin scattering experiment.

If one wants to determine the barrier distribution function from the mechanical damping at different frequencies, one often has to compare measurements of different elastic constants. A torsion pendulum measurement provides immediately the real and imaginary part of the shear modulus $G$. The vibrating reed technique measures Youngs modulus $Y$. In terms of the bulk modulus $B$, the inverse of the compressibility, Youngs modulus reads

$$
Y=\frac{9 B G}{3 B+G} .
$$

Since that quotient is close to $3 G$, it is a reasonable approximation to identify the $\tan \delta=Y^{\prime} / Y^{\prime \prime}$ results of the vibrating reed technique (in that technique often denoted as $Q^{-1}$ $=\tan \delta$ ) with that of the shear modulus $G$. The real part of the shear modulus $G^{\prime}$ can then be estimated to be $Y^{\prime} / 3$, or calculated accurately from the above equation if the bulk modulus is known.

A less safe connection is to longitudinal sound measurements, which determine the real and imaginary part of the elastic constant $c_{11}$, sometimes also denoted as the modulus $M$. In terms of $B$ and $G, c_{11}$ reads

$$
c_{11}=B+\frac{4}{3} G .
$$

Both contributions are of comparable size. Therefore, if one wants to determine $\tan \delta$ for the shear modulus from longitudinal sound attenuation data, one has to know the relative size of the two damping contributions from compression and from shear. Here it should be noted that the mechanical damping at very low temperatures is quite similar for transverse and longitudinal phonons in many glasses. ${ }^{27}$ As long as this holds, one can take $\tan \delta$ from the inverse mean-free path $l^{-1}$ of the longitudinal sound wave according to

$$
\tan \delta=\frac{v l^{-1}}{\omega},
$$

where $v$ is the sound velocity. In this equation, the mean-free path is defined as the distance where the sound-wave energy decreases by $1 / e$.

It is better to use data from transverse sound waves; there, the damping relates directly to the shear. However, in particular for light scattering Brillouin experiments, it is much easier to determine the damping for the longitudinal waves,

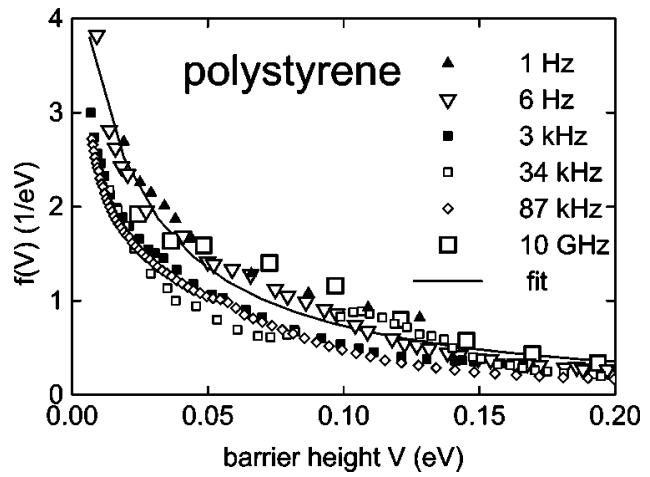

FIG. 3. Secondary barrier distribution function $f_{s}(V)$ calculated from literature data of the mechanical damping of amorphous polystyrene in the glass phase at different frequencies. For references see text. The line is a fit; the same fit is also shown in Figs. 4, 5, and 6.

which provide a much clearer signal. That damping is often given in terms of the half width at half maximum $\Gamma$ of the Brillouin line. Then

$$
\tan \delta=\frac{2 \Gamma}{\omega},
$$

where $\omega$ is the frequency of the Brillouin line.

Finally, one problem of the determination of $f(V)$ is to know $G$ at the temperature of the measurement; usually one has only $G^{\prime}$ and $G^{\prime \prime}$. A way out of this problem is to start at low temperatures, where $G \approx G^{\prime}$, determine $f(V)$ via Eq. (27), and then pursue $G^{\prime} / G$ to higher temperatures via Eq. (26). This way was followed throughout in this paper, at least as far as the determination of the secondary relaxation was concerned (the primary relaxation is too sharp to use the crude approximation of Eq. (27); there, one has to use the exact equations).

One can try to check the $G(T)$ values by Brillouin light scattering measurements of the transverse sound waves, usually at frequencies around $10 \mathrm{GHz}$. Even there, one has to reckon with some influence from the low-barrier part of the relaxations; this is, however, only a small correction which can be easily done if one has $f(V)$, using Eq. (26).

\section{B. Polystyrene: Secondary relaxation}

We begin the comparison to experiment with a heavily studied glass former, atactic polystyrene, one of the most fragile substances, ${ }^{5}$ an amorphous polymer where one can rely on a large number of experimental data, both at low temperatures and at the glass transition.

The low-temperature data were evaluated for temperatures above $10 \mathrm{~K}$; at that limiting temperature, one can begin to reckon with the validity of the Kramers picture. ${ }^{4}$ Figure 3 shows a compilation of many mechanical low-temperature data: torsion pendulum data at $1 \mathrm{~Hz},{ }^{28}$ at $6 \mathrm{~Hz},{ }^{29}$ vibrating reed data at 3, 34, and $87 \mathrm{kHz},{ }^{30-32}$ and Brillouin damping of longitudinal sound waves at $10 \mathrm{GHz}{ }^{33}$ The data cannot be said to coincide perfectly in this Gilroy-Phillips evaluation; nevertheless, the agreement is fair enough to support the 


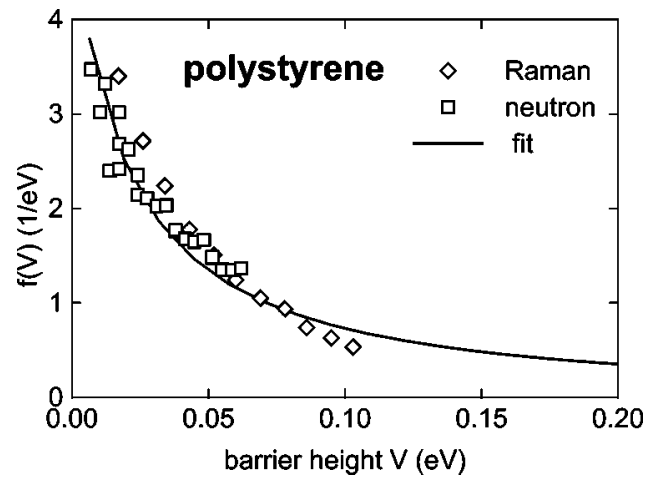

FIG. 4. Secondary barrier distribution function $f_{s}(V)$ calculated from literature data of neutron and Raman scattering from amorphous polystyrene in the glass phase. For references see text.

concept of a constant number density of uncoupled relaxation centers. There is no systematic variation with frequency; one rather has the impression that the differences stem from the different sample preparation of these seven measurements. The line in Fig. 3 represents the fit of $f_{s}(V)$ to these mechanical data. It is seen that $f_{s}(V)$ rises towards low barriers, as one would expect from the soft-potential Eq. (43). But the fit line falls below the soft-potential expectation already at rather low barriers, similar to observations in other glasses. ${ }^{15}$ In fact, spectral hole burning experiments ${ }^{34}$ between 4 and $80 \mathrm{~K}$ on six glasses including polystyrene revealed a $f(V) \sim V^{-1 / 2}$-behavior rather than the $f(V) \sim V^{-1 / 4}$-expectation of the soft-potential Eq. (43).

Figure 4 compares the same fit to the evaluation of Raman $^{16}$ and neutron ${ }^{35}$ data in terms of Eq. (41). In both cases, the data had to be adapted by an appropriate multiplication factor. In the neutron case, this multiplication factor turned out to be a factor of 1.6 smaller than the one calculated from Eq. (44) and the soft-potential fit parameters of polystyrene. ${ }^{15}$ One can rationalize this difference in terms of a slightly larger jump distance of the protons in the lowbarrier relaxational jumps; details will be given in a forthcoming publication combining time-of-flight and backscattering data of polystyrene. ${ }^{35}$

The good agreement between data points and the mechanical data fit line in Fig. 4 corroborates the earlier conclusion $^{16}$ of a temperature independent $f_{s}(V)$ in the glass phase of polystyrene. Note that the earlier conclusion did not stem from a comparison of Raman and mechanical data, but rather from a comparison of Raman data at three different temperatures, namely 100,200 , and $300 \mathrm{~K}$. The two different ways to check the temperature behavior of $f_{s}(V)$ in the glass phase provide the same result.

This temperature independence, however, no longer holds in the undercooled liquid phase, above the glass temperature of $372 \mathrm{~K}$ of polystyrene. Figure 5 shows neutron ${ }^{35}$ and longitudinal sound-wave damping data from the Brillouin technique $^{36}$ above $T_{g}$. In order to relate to the preceding figures, $f(V)$ is again plotted against the barrier height $V$. This implies that one sees the onset of the increase of $f_{s}(V)$ with increasing temperature at different values of $V$ in the two techniques, at $0.064 \mathrm{eV}$ for the neutrons and at $0.163 \mathrm{eV}$

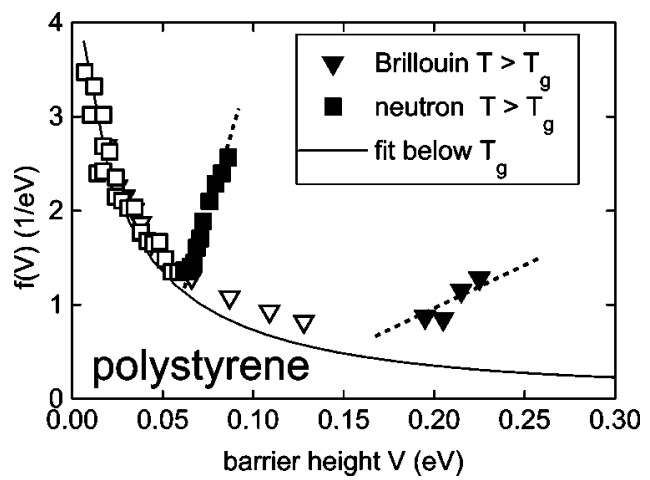

FIG. 5. Secondary barrier distribution function $f_{s}(V)$ calculated from literature data of neutron and Brillouin data of amorphous polystyrene, both in the glass phase and above the glass temperature $T_{g}$. For references see text.

for the Brillouin data. Note that in both cases the frequency is too high to see the $\alpha$ process at the temperatures of the measurement.

We conclude that $f_{s}(V)$ does indeed increase above $T_{g}$, as postulated above on the basis of the sum rule for $f(V)$, Eq. (15), and on the basis of the temperature dependence of the $\alpha$ process. The rise of $f_{s}(V)$ above $T_{g}$ can be characterized by the linear relation Eq. (31) with $\alpha_{s}=5 \pm 1$ for the neutron data and $\alpha_{s}=8 \pm 3$ for the Brillouin data (in the latter case, the large error is due to the small number of points and the insecurity of the value at $T_{g}$ ), within experimental error the same temperature coefficient for both sets of data.

Figure 6 shows $f_{s}(V)$ for polystyrene over the whole barrier range, together with the results of a torsion pendulum measurement at $1 \mathrm{~Hz}$ up to $T_{g},{ }^{28}$ an ultrasonic measurement just below $T_{g}$ (Ref. 37) and the neutron result up to $T_{g} .{ }^{35}$ The shaded area represents $f_{s}(V)$; the $\alpha$ peak above $1 \mathrm{eV}$ will be discussed in the next subsection.

Note the slight rise of $f_{s}(V)$ towards the $\alpha$ peak. Together with the cutoff of the secondary relaxation by the $\alpha$-peak, this appears as a small secondary relaxation peak just below the glass transition. In the literature, ${ }^{6,19}$ the first secondary relaxation peak which one finds below $T_{g}$ has the name Johari-Goldstein or slow- $\beta$ relaxation. There are many cases where it is much more pronounced than in polystyrene. ${ }^{38}$

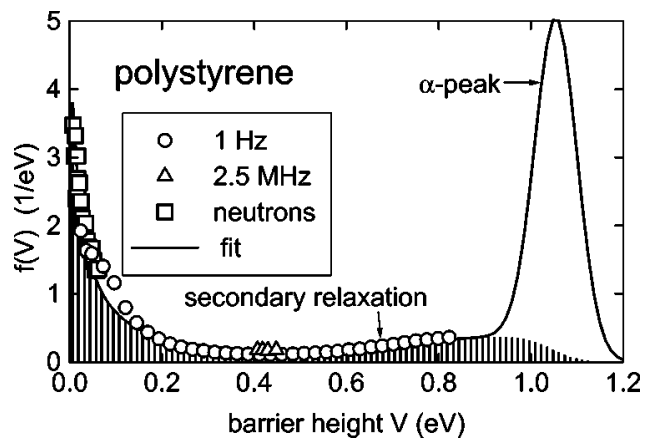

FIG. 6. Secondary barrier distribution function $f_{s}(V)$ calculated from literature mechanical damping and neutron data of amorphous polystyrene up to the glass transition. The peak at the end shows the Gaussian $f_{\alpha}(V)$ describing the $\alpha$-process at the glass temperature $T_{g}$. For references see text. 


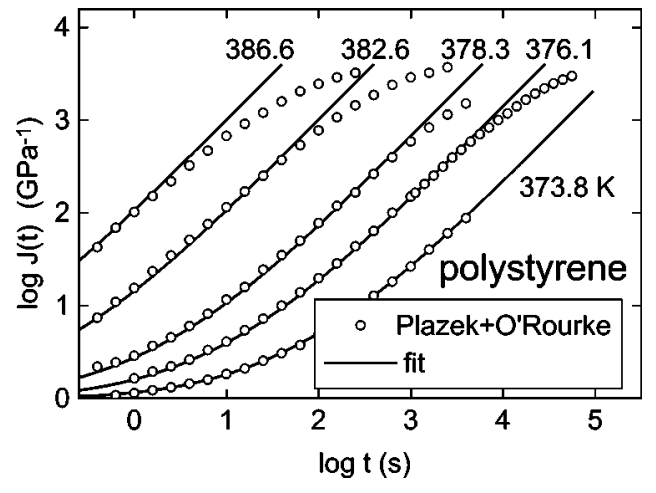

FIG. 7. Creep data of the $\alpha$ process in polystyrene at the glass transition (for references see text), together with a fit in terms of $f(V)$.

\section{Polystyrene: Glass transition}

The $\alpha$ process is characterized by the position $V_{\alpha}(T)$, the full width at half maximum $\Delta_{\alpha}$ of $f_{\alpha}(V)$ and the weight $w_{\alpha}$. The latter two should be temperature independent. Let us define $V_{\alpha}\left(T_{g}\right)=15 k_{B} T_{g} \ln (10)$ in order to have the corresponding relaxation time at $100 \mathrm{~s}$, and let us choose a Gaussian for the primary barrier distribution function $f_{\alpha}(V)$.

If one knows $f_{s}(V)$ from measurements in the glass phase, $w_{\alpha}$ can be calculated from the sum rule Eq. (30). In the case of polystyrene, one finds $w_{\alpha}=0.54$ for the fit function in Fig. 6.

The width $\Delta_{\alpha}=0.107 \mathrm{eV}$, the glass temperature $T_{g}$ $=372 \mathrm{~K}$ and the coefficient $\alpha_{s}=6.9 \pm 0.1$ was fitted to creep data $^{39}$ of polystyrene with a molecular weight of 600000 $\mathrm{g} / \mathrm{mol}$, data which are also treated in Ferry's book ${ }^{21}$ and which are shown in Fig. 7. The fit requires as an additional parameter the infinite frequency modulus $G=1.69 \mathrm{GPa}$ at $373.8 \mathrm{~K}$. Together with the low-temperature data and the known density variation of polystyrene with temperature, ${ }^{40}$ one deduces a Grüneisen $\Gamma_{g}=3.8$ for $G$ in polystyrene. With this value, one can also calculate $G$ for higher temperatures. The creep function $J(t)$ is obtained by first calculating $l(V)$ via Eq. (22), the viscosity from Eq. (19) and finally $J(t)$ from Eq. (17).

As seen in Fig. 7, one can describe the temperature shift of the $\alpha$ process with an appropriate rise of $f_{s}(V)$ above $T_{g}$. The sum rule Eq. (30) forces a temperature shift of $V_{\alpha}(T)$, which in turn provides the experimentally observed shift factors towards higher temperatures.

The scheme works quite well up to three decades in compliance; for still higher compliances, one gets into the plateau regime from the chain entanglement, ${ }^{21}$ which is beyond the present considerations.

\section{Vitreous silica and sodium silicate}

Vitreous silica is the case for which the Gilroy-Phillips idea $^{11}$ was originally developed. The compatibility between mechanical relaxation around $20 \mathrm{~K}$ and inelastic neutron scattering at room temperature was demonstrated seven years later. ${ }^{18} \mathrm{~A}$ very recent Raman experiment ${ }^{17}$ showed the temperature independence of $f_{s}(V)$ up to room temperature, to-

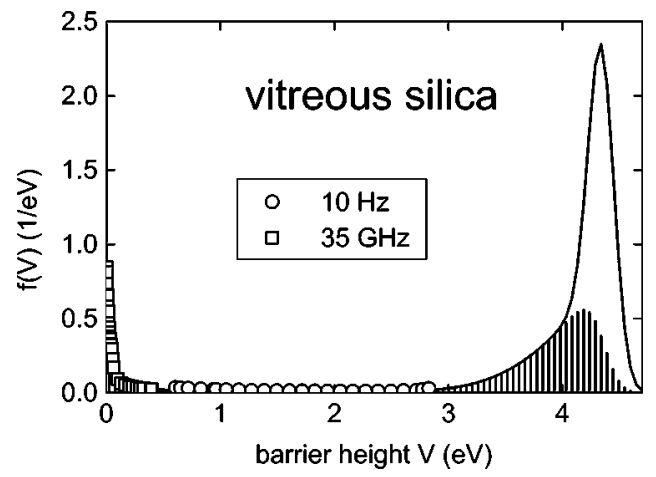

FIG. 8. The barrier distribution function of vitreous silica, together with torsion pendulum and Brillouin damping data. For references see text.

gether with an excellent agreement of the shape of the function from mechanical data with the one from Raman data.

Of the many low-barrier mechanical data in the literature, we include here merely one of the Brillouin damping experiments at $35 \mathrm{GHz}{ }^{41}$ The range of higher barriers is covered by a torsion pendulum measurement $\mathrm{t}^{42}$ and a $\mathrm{mHz}$ experiment at the glass transition itself. ${ }^{43}$ Figure 8 shows the barrier distribution function fitting these data over the whole range. Again, it turns out to be possible to fit the $\alpha$ relaxation in terms of a Gaussian for the primary barrier distribution function $f_{\alpha}(V)$, ascribing the slow rise of the damping towards the glass transition to secondary relaxation (the shaded area in Fig. 8). Note that in the silica case the assignment of secondary and primary relaxation is by no means as clear as in the preceding polystyrene case; by choosing a Gaussian for $f_{\alpha}(V)$ one enforces the existence of a large JohariGoldstein-like secondary relaxation peak just below the glass transition. But it is not a peak, but rather a rise towards higher barriers cut off by the glass transition.

Figure 9 shows the fit of the glass transition data, ${ }^{43}$ calculated with $T_{g}=1460 \mathrm{~K}, G=31 \mathrm{GPa}, w_{\alpha}=0.55$, and $\Delta_{\alpha}$ $=0.263 \mathrm{eV}$. If one takes the value $T_{g}-T_{0}=850 \mathrm{~K}$ and its rather large error bars from the shift factors determined in, ${ }^{43}$ one calculates $\alpha_{s}=6 \pm 4$ from Eq. (33).

It is interesting to compare vitreous silica to a sodium silicate glass, $\mathrm{Na}_{2} \mathrm{O}: 2 \mathrm{SiO}_{2}$, which has a much lower glass temperature $T_{g}=733 \mathrm{~K}$ and is much more fragile, ${ }^{43}$ with

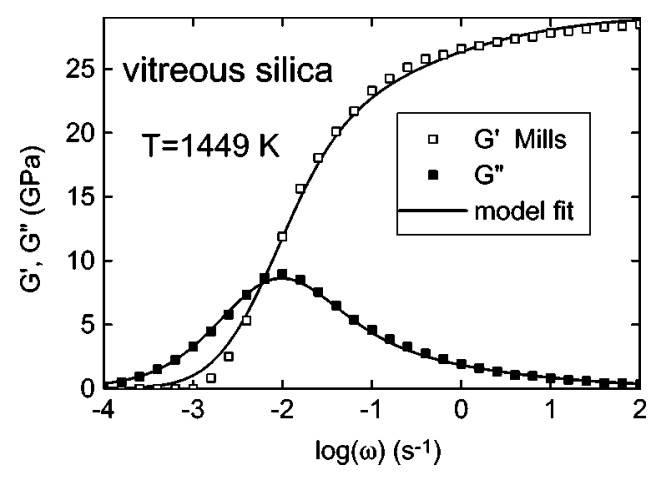

FIG. 9. Dynamical mechanical data of vitreous silica at the glass transition, together with the model fit (see text). 


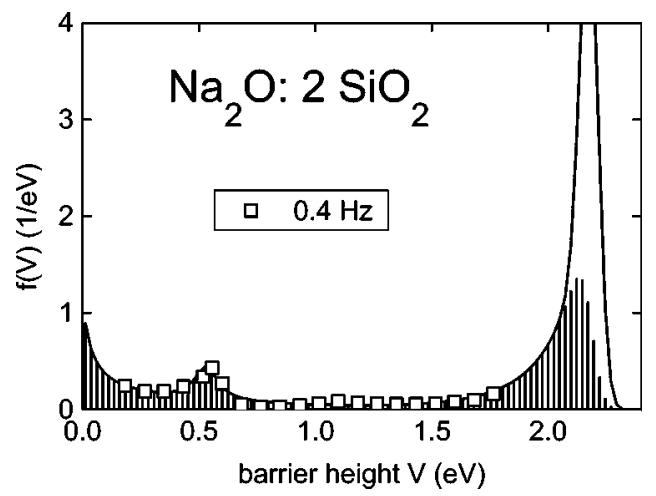

FIG. 10. The barrier distribution function of $\mathrm{Na}_{2} \mathrm{O}: 2 \mathrm{SiO}_{2}$, together with torsion pendulum data. For references see text.

$T_{g}-T_{0}=267 \mathrm{~K}$. Figure 10 shows the barrier distribution function, together with torsion pendulum data ${ }^{44}$ at $0.4 \mathrm{~Hz}$. The $\alpha$-peak region is again fitted to the $\mathrm{mHz}$ data, ${ }^{43}$ shown in Fig. 11, with $G=19 \mathrm{GPa}, w_{\alpha}=0.39$, and $\Delta_{\alpha}$ $=0.092 \mathrm{eV}$. The shift factors ${ }^{43}$ require $\alpha_{s}=13 \pm 1.5$.

\section{RESULTS AND DISCUSSION}

\section{A. Results for the flow process}

The preceding section showed for all three examples a good fit of the $\alpha$ relaxation in terms of a narrow Gaussian primary barrier distribution function, together with a bigger or smaller contribution of the secondary relaxation. The contribution of the secondary relaxation at the $\alpha$ process was biggest in the strong glass former silica and smallest in the very fragile case of polystyrene. In all three cases, excellent fits of the time or frequency dependence of the $\alpha$ process could be obtained (see Figs. 7, 9, and 11). Simultaneously, the temperature dependence of the shift factors for $\tau_{\alpha}$ could be described accurately by the temperature slope $\alpha_{s}$ of the rise of the secondary relaxation above $T_{g}$ in Eq. (31).

Table I compiles the fit parameters for the $\alpha$ process determined in the preceding section.

In all three cases, the relative weight $w_{\alpha}$ of the $\alpha$ process is not very far from $1 / 2$. The $\alpha$ process seems to occur when the secondary relaxations reduce the shear modulus to about half its infinite frequency value, i.e., when the secondary

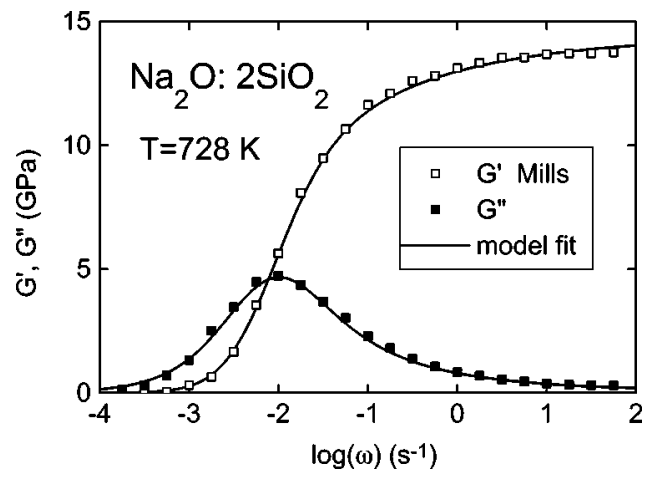

FIG. 11. Dynamical mechanical data of $\mathrm{Na}_{2} \mathrm{O}: 2 \mathrm{SiO}_{2}$ at the glass transition, together with the model fit (see text).
TABLE I. Model parameters for the $\alpha$ process.

\begin{tabular}{lccc}
\hline \hline Substance & Polystyrene & $\mathrm{SiO}_{2}$ & $\mathrm{Na}_{2} \mathrm{O}: 2 \mathrm{SiO}_{2}$ \\
\hline$T_{g}(\mathrm{~K})$ & 372 & 1460 & 733 \\
$G\left(T_{g}\right)(\mathrm{GPa})$ & 1.69 & 31 & 19 \\
$w_{\alpha}$ & 0.54 & 0.54 & 0.39 \\
$\Delta_{\alpha}(\mathrm{eV})$ & 0.107 & 0.263 & 0.092 \\
$\alpha_{s}$ & $6.9 \pm 0.1$ & $6 \pm 4$ & $13 \pm 1.5$ \\
\hline \hline
\end{tabular}

relaxational shear response equals the elastic one. The situation reminds one of the definition of the Maxwell time $\tau_{M}$ $=\eta_{0} / G$, the time when the flow response equals the elastic one. Thus one could think of a generalized Maxwell criterion for the onset of the flow process, namely when the shear response by secondary relaxation is the same as the elastic shear response. Intuitively, this is plausible: the time at which a macroscopic shear stress for a given strain decays to half its initial value should correspond to the lifetime of the microscopic shear pattern of the glass former, because the microscopic stresses will be expected to decay on the same time scale as the macroscopic ones. But a decay of the microscopic stresses necessarily implies a coupling between different relaxation centers in the glass. Thus one would have to go beyond the simple idea of uncoupled relaxation centers to understand the flow process. ${ }^{45}$

In all three cases, the width $\Delta_{\alpha}$ is relatively narrow, less than one tenth of the barrier $V_{\alpha}\left(T_{g}\right)$ itself, in the third case even less than the twentieth part.

Finally, the coefficient $\alpha_{s}$ for the temperature rise of the secondary barrier distribution function $f_{s}(V)$ above $T_{g}$, related to the fragility of the glass former, is not so very different for the three cases. There is a factor of two between silica and the silicate glass, but taking the differences in the glass temperature into account, one sees that the relative rise per Kelvin is the same for the two glasses, admittedly within large error bars. The difference is rather in $f_{s}(V)$ itself; obviously, relaxing entities form much more easily and with much lower barriers in the multiply broken network of the sodium silicate glass than in the continuous random network of silica, making the glass temperature a factor of two lower.

The large difference in fragility between silica and polystyrene is not so much due to a difference in $\alpha_{s}$, but rather in the product $V f_{s}(V)$ at $T_{g}$ (see Figs. 6 and 8), which is about 0.3 in polystyrene and about 2.5 in silica. This product enters into Eq. (33) for the Vogel-Fulcher temperature $T_{0}$. In this view, a substance is fragile when it has a low density of secondary relaxing entities at the relaxation time of the flow process. Naturally, the fast rise of the number of secondary relaxing entities above $T_{g}$ remains a necessary condition.

The coefficient $\alpha_{s}=6.9 \pm 0.1$ for polystyrene in Table I, obtained from the temperature dependence of the shift factors (see Fig. 7), agrees reasonably well with the values $\alpha_{s}$ $=5 \pm 1$ and $\alpha_{s}=8 \pm 3$ determined from neutron and Brillouin data above $T_{g}$ (see Fig. 5). It need not be exactly the same, because the coefficient $\alpha_{s}$ could still depend on the barrier height $V$. 


\section{B. Discussion}

Here is the proper place to remind the reader that with all the presented equations and fits, the glass transition is not really explained. The Gilroy-Phillips formalism supplies only a reformulation of well-known equations in terms of a barrier distribution function. It is a different way to look at the data, an encouragement to seek explanations in a different direction, complementary to attempts to understand the glass transition from the liquid side, ${ }^{46}$ because it starts from a description of the relaxation in the glass phase. But the present work does neither explain the fast rise of the secondary relaxation above $T_{g}$, nor does it supply a quantitative explanation why the shear modulus breaks down completely when it is halved by the secondary relaxation. It merely helps to quantify and to visualize these experimental facts.

The concept of the barrier distribution function is based on the idea of independent thermally activated relaxation processes in disordered surroundings. As long as this idea applies without any restriction, one should find a temperature-independent barrier distribution function, as one does indeed in the glass phase of polystyrene and silica. The quantitative comparison of measurements at different temperatures and frequencies with the equations of Sec. II C enables a much more stringent check of the Arrhenius concept than a mere Arrhenius temperature shift of a broad relaxation peak with frequency. ${ }^{1-3}$ From the few such stringent checks reported so far, it is already clear that the concept does not always work perfectly well in the glass phase. In BPA-PC (amorphous bisphenol A polycarbonate), another amorphous polymer, there is Raman evidence ${ }^{16}$ for an increase of the secondary barrier distribution function $f_{s}(V)$ with temperature below $T_{g}$, even though the $\gamma$-relaxation peak shift is perfectly Arrhenius-like. ${ }^{47}$ There seem to be more such cases. $^{48}$ This is yet another question calling for a closer investigation.

The outcome of the fits of the flow process in terms of the primary barrier distribution function $f_{\alpha}(V)$ shows once more the collectivity of the flow process. These are clearly not independent thermally activated relaxation processes; otherwise $V_{\alpha}$ would not shift with temperature. There has been an attempt ${ }^{49}$ to explain the temperature dependence of $V_{\alpha}(T)$ in terms of a proportionality to $G(T)$ ("flow by shoving"). However, as pointed out in Sec. III C, one finds a Grüneisen $\Gamma_{g}=3.8$ for $G(T)$ in polystyrene. This is a factor of seven too weak to explain the temperature shift of $V_{\alpha}(T)$.

In any case, the description of the flow process in terms of $f_{\alpha}(V)$ in principle does not prejudice anything. One can always return from $f_{\alpha}(V)$ to the conventional description ${ }^{21}$ in terms of $H(\tau)$ via Eq. (11). What might be questionable is the specific subdivision into primary and secondary relaxation. Here, it was decided to fit the primary barrier distribution function $f_{\alpha}(V)$ with a Gaussian. On the basis of the data, this choice can be only justified for polystyrene; in the other two cases, one could choose differently. The conclusion that the shear modulus is reduced to about half its infinite frequency value by the secondary relaxation alone would then no longer be valid for these two cases. In this context, it would be interesting to evaluate glasses with a more pronounced Johari-Goldstein peak, to see how well the concept works in those cases.

Independent of the choice of a specific function for the primary barrier distribution function, the new description is much more convenient than the old one if one wants to compare different temperatures. It does not only allow for a stringent check of the Arrhenius behavior in the glass phase, but it also holds the promise to provide a deeper understanding of the glass transition riddles. ${ }^{6,5}$ As shown in Sec. II E, the stretching of the flow process is quantitatively related to the width of the $\alpha$-peak. More important, the Vogel-Fulcher behavior is an inherent feature of the generic case of a constant secondary barrier distribution function, provided it has a constant temperature rise. But one can as well understand deviations ${ }^{50,51}$ from the Vogel-Fulcher behavior in terms of maxima and minima of the secondary barrier distribution function. In particular, one must expect deviations from a single Vogel-Fulcher law when $V_{\alpha}(T)$ sweeps through a secondary relaxation peak, as one indeed observes. ${ }^{52}$

\section{SUMMARY AND CONCLUSIONS}

The Gilroy-Phillips model ${ }^{11}$ for secondary relaxation in glasses, based on the Arrhenius-Kramers ${ }^{4}$ picture of thermally activated jumps over energy barriers, has been extended to describe the flow process (primary or $\alpha$ process). The proper treatment of uncoupled thermally activated relaxation events in disordered surroundings yields a temperatureindependent barrier distribution function $f(V)$. The temperature independence holds as long as one has a constant number of independent relaxation centers. The function has to integrate into 1 in order to bring the shear modulus down to zero, a very convenient sum rule. As it turns out, one can use the formalism to describe any kind of relaxation, admitting a temperature dependence of the barrier distribution function. Thus one can separate the trivial Arrhenius temperature dependence from nontrivial temperature changes of the relaxation.

The relaxation below the glass temperature $T_{g}$, where the dynamics is restricted to local back-and-forth jumps, should be describable in terms of a temperature-independent barrier distribution function [in some cases like polystyrene, CKN (a mixed calcium-potassium nitrate glass), and silica ${ }^{16,17}$ it works; in others it does not $\left.{ }^{16,48}\right]$, but the flow process is most certainly of a different kind. Therefore one has to postulate a secondary barrier distribution function $f_{s}(V)$ for the secondary relaxation, and an additional primary barrier distribution function $f_{\alpha}(V)$ for the flow process. This additional primary function shifts its maximum to lower values with increasing temperature and serves simultaneously as a relatively sharp cutoff for the secondary relaxation. This property supplies a quantitative basis for the puzzling relation between the fragility and the fast rise of the picosecond process above $T_{g} .{ }^{19}$ It explains the unusual temperature dependence of the flow process in terms of the temperature rise of the secondary relaxation, and might even shed some light on the detailed temperature dependence of the flow process in specific substances. ${ }^{50-52}$ 
Reformulating the classical rheological equations, ${ }^{21}$ experimental literature data in polystyrene, vitreous silica and a sodium silicate glass could be fitted in terms of the barrier distribution functions. The results corroborate the earlier conclusion ${ }^{16}$ of a temperature-independent secondary barrier distribution function in the glass phase of polystyrene, but show a strong increase of the secondary relaxation with increasing temperature above $T_{g}$. Taking the flow process as a Gaussian barrier distribution function, the fits show the onset of the flow process more or less at the point where the secondary relaxation reduces the shear modulus to half its infinite frequency value. This was not only found in polystyrene, but in the two other cases as well. It remains to be seen whether such a generalized Maxwell criterion for the onset of the flow process is a general property of glass formers. In polystyrene, the temperature coefficient of the fast rise of $f_{s}(V)$ above $T_{g}$ calculated from the fragility coincided rea- sonably well with the fast rise seen in neutron and Brillouin experiments.

The findings suggest a rapid change of the sampled energy landscape above $T_{g}$, the system visiting regions with more and more minima and saddle points as the temperature rises, thus also explaining the entropy crisis at the Kauzmann temperature. This does not really answer the central questions around the glass transition, but focuses the attention on two points: (i) what is the possible mechanism of formation of an increasing number of secondary relaxation entities with increasing temperature above $T_{g}$ ? (ii) Why does the flow process set in when the integrated secondary relaxational response to an external shear stress equals the elastic one?

\section{ACKNOWLEDGMENTS}

Helpful discussions with A. Wischnewski, E. W. Fischer, K. L. Ngai, and W. Kob are gratefully acknowledged.
${ }^{1}$ S. Hunklinger and W. Arnold, in Physical Acoustics, Vol. 12, edited by R. N. Thurston and W. P. Mason (Academic Press, New York, 1976), p. 277.

${ }^{2}$ S. Hunklinger and M. v. Schickfus, in Amorphous Solids: Low Temperature Properties, edited by W. A. Phillips (Springer, Berlin, 1981), p. 81

${ }^{3} \mathrm{~J}$. Heijboer, in Molecular basis of transitions and relaxations, edited by D. J. Meier (Gordon and Breach, London, 1978), p. 75

${ }^{4}$ H. A. Kramers, Physica (Utrecht) 7, 284 (1940).

${ }^{5}$ R. Böhmer, K. L. Ngai, C. A. Angell, and D. J. Plazek, J. Chem. Phys. 99, 4201 (1993).

${ }^{6}$ M. D. Ediger, C. A. Angell, and S. R. Nagel, J. Phys. Chem. 100, 13200 (1996).

${ }^{7}$ H. Fröhlich, Theory of Dielectrics (Oxford University Press, Oxford, 1949).

${ }^{8}$ J. D. Hoffman and H. G. Pfeiffer, J. Chem. Phys. 22, 132 (1954).

${ }^{9}$ W. A. Phillips, J. Low Temp. Phys. 7, 351 (1972); P. W. Anderson, B. I. Halperin, and C. M. Varma, Philos. Mag. 25, 1 (1972).

${ }^{10}$ M. Pollak and G. E. Pike, Phys. Rev. Lett. 28, 1449 (1972).

${ }^{11}$ K. S. Gilroy and W. A. Phillips, Philos. Mag. B 43, 735 (1981).

${ }^{12}$ D. A. Parshin, Phys. Solid State 36, 991 (1994).

${ }^{13}$ R. Keil, G. Kasper, and S. Hunklinger, J. Non-Cryst. Solids 164-166, 1183 (1993).

${ }^{14}$ D. Tielbürger, R. Merz, R. Ehrenfels, and S. Hunklinger, Phys. Rev. B 45, 2750 (1992).

${ }^{15}$ M. A. Ramos and U. Buchenau, Phys. Rev. B 55, 5749 (1997).

${ }^{16}$ N. V. Surovtsev, J. Wiedersich, V. N. Novikov, E. Rössler, and A. P. Sokolov, Phys. Rev. B 58, 14888 (1998).

${ }^{17}$ J. Wiedersich, S. V. Adichtchev, and E. Rössler, Phys. Rev. Lett. 84, 2718 (2000).

${ }^{18}$ U. Buchenau, H. M. Zhou, N. Nücker, K. S. Gilroy, and W. A. Phillips, Phys. Rev. Lett. 60, 1318 (1988).

${ }^{19}$ C. A. Angell, Science 267, 1924 (1995).

${ }^{20} \mathrm{~T}$. Mura, Micromechanics of Defects in Solids (Martinus Nijhoff, The Hague 1982), Chap 2, p. $63 \mathrm{ff}$.
${ }^{21}$ D. J. Ferry, Viscoelastic Properties of Polymers, 3rd ed. (Wiley, New York 1980).

${ }^{22}$ C. A. Angell and K. J. Rao, J. Chem. Phys. 57, 470 (1972).

${ }^{23}$ G. Adam and J. H. Gibbs, J. Chem. Phys. 43, 139 (1965).

${ }^{24}$ W. Marshall and S. W. Lovesey, Theory of Thermal Neutron Scattering (Oxford, Clarendon Press, 1971), p. 65 ff.

${ }^{25}$ J. Wuttke, J. Hernandez, G. Li, G. Coddens, H. Z. Cummins, F. Fujara, W. Petry, and H. Sillescu, Phys. Rev. Lett. 72, 3052 (1994).

${ }^{26}$ A. P. Sokolov, U. Buchenau, W. Steffen, B. Frick, and A. Wischnewski, Phys. Rev. B 52, R9815 (1995).

${ }^{27}$ J. F. Berret and M. Meissner, Z. Phys. B: Condens. Matter 70, 65 (1988).

${ }^{28}$ F. R. Schwarzl, Polymermechanik (Springer, New York 1990), Fig. 9.16.

${ }^{29}$ K. M. Sinnott, Soc. Plastics Engrs. Trans. 2, 65 (1962).

${ }^{30}$ A. Nittke, M. Scherl, P. Esquinazi, W. Lorenz, Li Yunyun, and F. Pobell, J. Low Temp. Phys. 98, 517 (1995).

${ }^{31}$ O. Yano and Y. Wada, J. Polym. Sci. A-2 9, 669 (1971).

${ }^{32}$ K. A. Topp and D. G. Cahill, Z. Phys. B: Condens. Matter 101, 235 (1996).

${ }^{33}$ A. P. Sokolov, V. N. Novikov, and B. Strube, Europhys. Lett. 38, 49 (1997).

${ }^{34}$ W. Köhler and J. Friedrich, Phys. Rev. Lett. 59, 2199 (1987).

${ }^{35}$ S. Koizumi, A. Paciaroni, and U. Buchenau (unpublished).

${ }^{36}$ G. D. Patterson, J. Polym. Sci., Part B: Polym. Phys. 15, 579 (1977).

${ }^{37}$ A. Sahnoune, F. Massines, and L. Piché, J. Polym. Sci., Part B: Polym. Phys. 34, 341 (1996).

${ }^{38}$ G. P. Johari and M. J. Goldstein, J. Chem. Phys. 53, 2372 (1970); 55, 4245 (1971).

${ }^{39}$ D. J. Plazek and V. M. O'Rourke, J. Polym. Sci. A-2 9, 209 (1971).

${ }^{40}$ F. R. Schwarzl, Polymermechanik (Springer, New York, 1990), Fig. 6.12.

${ }^{41}$ R. Vacher, J. Pelous, F. Plicque, and A. Zarembowitch, J. NonCryst. Solids 45, 397 (1981).

${ }^{42}$ R. Brückner, J. Non-Cryst. Solids 5, 123 (1970). 
${ }^{43}$ J. J. Mills, J. Non-Cryst. Solids 14, 255 (1974).

${ }^{44}$ P. W. L. Graham and G. E. Rindone, Phys. Chem. Glasses 8, 160 (1967).

${ }^{45}$ K. L. Ngai, G. N. Greaves, and C. T. Moynihan, Phys. Rev. Lett. 80, 1018 (1998).

${ }^{46}$ W. Götze and L. Sjögren, Rep. Prog. Phys. 55, 241 (1992).

${ }^{47}$ G. Floudas, J. S. Higgins, G. Meier, F. Kremer, and E. W. Fischer, Macromolecules 26, 1676 (1993).

${ }^{48}$ A. P. Sokolov (private communication).
${ }^{49}$ N. B. Olsen, J. C. Dyre, and T. Christensen, Phys. Rev. Lett. 81, 1031 (1998).

${ }^{50}$ F. Stickel, E. W. Fischer, and R. Richert, J. Chem. Phys. 102, 6251 (1995).

${ }^{51}$ F. Stickel, E. W. Fischer, and R. Richert, J. Chem. Phys. 104, 2043 (1996).

${ }^{52}$ C. Hansen, F. Stickel, T. Berger, R. Richert, and E. W. Fischer, J. Chem. Phys. 107, 1086 (1997). 